\title{
Modeling Dynamic Effects of Promotion on Interpurchase Times
}

\author{
Dennis Fok* \\ Econometric Institute \\ Richard Paap \\ Erasmus University Rotterdam Erasmus University Rotterdam
}

\author{
Philip Hans Franses \\ Econometric Institute \\ Department of Marketing and Organization \\ Erasmus University Rotterdam
}

Econometric Institute Report EI 2002/37

\begin{abstract}
In this paper we put forward a duration model to analyze the dynamic effects of marketing-mix variables on interpurchase times. We extend the accelerated failuretime model with an autoregressive structure. An important feature of our model is that it allows for different long-run and short-run effects of marketing-mix variables on interpurchase times. As marketing efforts usually change during the spells, we explicitly deal with time-varying covariates. Our empirical analysis of purchases in three different categories reveals that, for some segments of households, the shortrun effects of marketing-mix variables are significantly different from the long-run effects.
\end{abstract}

Key words: dynamic duration model, time-varying covariates, error-correction model, unobserved heterogeneity.

JEL codes: C41, C23

*We thank Teun Kloek for helpful comments. The models described in this paper are implemented in Ox 3.10 (Doornik 1999). We thank participants of Marketing Science 2002 and the Econometric Society European Meeting 2002 conferences for their comments. Detailed results of the empirical section are available from the first author. Address for correspondence: Dennis Fok, Erasmus University Rotterdam, Econometric Institute H11-2, P.O. Box 1738, NL-3000 DR Rotterdam, The Netherlands, e-mail: dfok@few.eur.nl 


\section{Introduction}

For marketing managers it is important to understand the dynamic effects of marketingmix variables like promotion and advertising on marketing performance measures such as sales, market shares and profitability. Particularly, it is relevant to understand the long-run effects of marketing efforts, as this knowledge can for example lead to more efficient marketing strategies. Examples of recent studies that address this issue are Mela et al. (1997), Dekimpe et al. (1999), Jedidi et al. (1999) and Paap and Franses (2000), to mention just a few. The literature contains two different approaches. One approach tries to capture the (long-run) effects of marketing instruments on for example the price elasticity (Mela et al. 1997, Jedidi et al. 1999). Dynamics are then incorporated through the responses to marketing instruments. The second approach, which is considered in the present paper, focuses on dynamic effects in behavior (Dekimpe et al. 1999, Paap and Franses 2000).

In this paper we address the issue of measuring the long-run and short-run impact of marketing-mix variables on interpurchase times, which is particularly relevant for retailers. The theoretical and empirical analysis of purchase-timing behavior of households has received considerable attention in the past and in recent years. The analysis of interpurchase timing can give interesting insights in household behavior. Purchase timing can be especially informative to learn about inventory management and consumption rate, in fact the purchase timing and the volume bought are the only two measures related to inventory that are usually available. Furthermore, we can study purchase acceleration and stock piling using interpurchase timing. Blattberg et al. (1981) show under stringent conditions that promotions lead to purchase acceleration. Empirical evidence for this behavior can be found in Gupta (1988) and Helsen and Schmittlein (1992) among others, although for example Neslin et al. (1985) report that promotions were less likely to accelerate purchase times. A review of interpurchase time modeling before 1990 can be found in Jain and Vilcassim (1991, Table 1).

More recently, researchers focus on using hazard functions to analyze the effect of 
promotions on interpurchase times, see among others Helsen and Schmittlein $(1992,1993)$, Jain and Vilcassim (1991), Vilcassim and Jain (1991), Gönül and Srinivasan (1993a) and Chintagunta and Prasad (1998). The last three studies also incorporate unobserved household heterogeneity. An important extension to modeling interpurchase times for two related product categories is given in Chintagunta and Haldar (1998).

Dynamic models for interpurchase times are relatively scarce, although one might expect strong dynamic effects in practice. For example, a promotion may shorten the present interpurchase time, while it likely lengthens future interpurchase times due to stock piling. An example of a study that explicitly incorporates dynamic structures in purchase timing is Allenby et al. (1999). In that paper, dynamics in durations are modeled by lagged interpurchase times, but no explicit separation of long-run from short-run effects of marketing mix variables is pursued. As we believe that such differences might exist, we aim to contribute to the literature by putting forward a dynamic model for interpurchase times that does allow for different long-run and short-run effects. The model extends the familiar accelerated failure-time model by including lagged interpurchase times as well as lagged covariates. Rewriting this model as an Error Correction Model [ECM] allows us to distinguish the long-run from the short-run effects, see Hendry et al. (1984).

The values of marketing mix-variables, like price and promotion, are likely to change during interpurchase spells. In most marketing applications of duration models, it is assumed that covariates remain constant during spells, which is perhaps imposed for convenience. In contrast, in this paper we follow a similar approach as Gupta (1991), that is, we allow for time-varying covariates in the hazard specification. Additionally, many studies have emphasized the relevance of unobserved household heterogeneity, and that it should be taken into account when analyzing purchase behavior. Therefore, we accommodate for unobserved differences across households by a latent class approach. In many studies, unobserved heterogeneity is incorporated using a mixed proportional hazard model, where one introduces a stochastic multiplicative factor to the hazard specification, see Lancaster (1979) and see Gönül and Srinivasan (1993a) for an application in marketing. In this paper we also allow for different effects of the covariates including the marketing 
mix on the interpurchase times, see also Vakratsas and Bass (2002) for a similar approach. Indeed, such heterogeneity may be especially relevant for modeling dynamics in purchase timing. For example, the population may contain households with very different dynamic purchase timing patterns.

In sum, we propose a dynamic model for interpurchase time, with possibly differing short-run and long-run effects of covariates, which incorporates unobserved heterogeneity and also takes care of time-varying covariates in between purchases.

The outline of our paper is as follows. In Section 2, we discuss our dynamic duration model. We show how the accelerated failure-time model can be extended to allow for time-varying covariates and possibly differing long-run and short-run effects of marketing variables. We discuss in detail how one can interpret the parameters and estimate them using maximum likelihood. In Section 3, we apply our model to purchases in three distinct categories of frequently purchased consumption goods, that is liquid laundry detergent, catsup and yogurt. One of our main empirical findings is that, for some household segments, the short-run effects of marketing mix variables are significantly different from the long-run effects. In Section 4, we conclude our paper with a discussion of the main results and with suggestions for further research topics.

\section{A Dynamic model for Interpurchase Times}

In this section we put forward our dynamic model for interpurchase times, which enables a separate evaluation of long-run and short-run effects of promotion and other marketingmix variables. In Section 2.1, we present the functional form of the hazard specification and discuss how we take care of time-varying covariates. In Section 2.2, we introduce autoregressive dynamics in our model. The interpretation of the dynamic structure is discussed in Section 2.3. Finally, in Section 2.4, we consider parameter estimation.

\subsection{Hazard Specification}

Assume that a household $i=1, \ldots, I$ purchases a certain product at time $d_{i, n}$, for $n=$ $0, \ldots, N_{i}$ over a certain period of time. The $N_{i}$ interpurchase times of this household are 
therefore defined by $t_{i, n}=d_{i, n}-d_{i, n-1}$ with $n=1, \ldots, N_{i}$. To model the interpurchase times, we consider a hazard specification. Denote the hazard corresponding to the $n$-th purchase decision of household $i$ by

$$
\lambda_{i, n}\left(t \mid x_{i, n}(t), \theta_{i}\right)
$$

where $x_{i, n}(t)$ denotes a vector of covariates explaining the hazard of household $i$ for the $n$-th purchase decision at time $t$ and $\theta_{i}$ is a household-specific parameter vector. The explanatory variables are a function of time $t$. In this paper, $t$ denotes the interpurchase time. For the $n$-th interpurchase spell the calendar time is given by $d_{i, n-1}+t$. Hence $x_{i, n}(t)$ gives the value of the covariates at calendar time $d_{i, n-1}+t$.

When modeling interpurchase times, it is unrealistic to assume that the covariates are constant during the spell, see Gupta (1991). In other words, it is unrealistic to assume that households do not notice these variations at no-purchase store visits. The set of covariates will usually include marketing instruments such as price and display. These variables do not evolve smoothly over time, rather, they usually change on a weekly basis or perhaps every day. Denote by $\tau_{l}$, for $l=0, . ., L$, the time indexes where there is a change in one of the covariates. For ease of exposition we assume that the covariates are constant within a week. Week 1 then corresponds to the time interval $\left[\tau_{0}, \tau_{1}\right]$. Denote by $K_{i, n}(t)$ the week number corresponding to $t$ time periods after the start of the $n$-th spell of household $i$. This week then starts at $\tau_{K_{i, n}(t)-1}$ and ends at $\tau_{K_{i, n}(t)}$. In Figure 1, we give a graphical representation of the purchase process. In this example we have purchases in weeks 2 and 4 , and in this case we would therefore have $K_{i, n}(0)=2$ and $K_{i, n}\left(t_{i, n}\right)=4$.

To derive the distribution of the interpurchase times we use the fact that the survivor function $\left.S_{i, n}\left(t \mid x_{i, n}(t), \theta_{i}\right)\right)$ equals $\exp \left(-\Lambda_{i, n}\left(t \mid x_{i, n}(t), \theta_{i}\right)\right)$, where $\Lambda_{i, n}\left(t \mid x_{i, n}(t), \theta_{i}\right)$ is the integrated hazard function. This function is defined as

$$
\Lambda_{i, n}\left(t \mid x_{i, n}(t), \theta_{i}\right)=\int_{0}^{t} \lambda_{i, n}\left(u \mid x_{i, n}(u), \theta_{i}\right) d u .
$$

Note that the integrated hazard function depends on the whole path of $x_{i, n}(u)$ for $u=$ $0, \ldots, t$, see Lancaster (1990) for a discussion. This integral can be decomposed by identifying intervals in which $x_{i, n}(t)$ is constant. We decompose the integral in three parts, 
that is, (i) from the start of the duration to the end of the corresponding week, (ii) the weeks completely contained in the duration, and (iii) from the start of the final week to the end of the duration. The integrated hazard can be decomposed as

$$
\begin{aligned}
& \Lambda_{i, n}\left(t \mid x_{i, n}(t), \theta_{i}\right)=\int_{0}^{\tau_{K_{i, n}(0)-d_{i, n-1}}} \lambda_{i, n}\left(u \mid x_{i, n}(u), \theta_{i}\right) d u \\
& \quad+\sum_{k=K_{i, n}(0)}^{K_{i, n}(t)-2} \int_{\tau_{k}-d_{i, n-1}}^{\tau_{k+1}-d_{i, n-1}} \lambda_{i, n}\left(u \mid x_{i, n}(u), \theta_{i}\right) d u+\int_{\tau_{K(t)-1}-d_{i, n-1}}^{t} \lambda_{i, n}\left(u \mid x_{i, n}(u), \theta_{i}\right) d u,
\end{aligned}
$$

see Gupta (1991) for a similar approach.

As the computation of the integrated hazard function is computationally intensive, it is convenient to have a closed-form expression for the individual elements of (3). Therefore, in this paper we use as starting point the hazard structure of an accelerated failure-time model with a log-logistic baseline hazard. This hazard specification leads to an analytical expression of the integrated baseline hazard, while it allows for a non-monotonic hazard function. Another advantage of using an accelerated failure-time specification is that it corresponds with a linear representation for the case of constant covariates during spells, see Kalbfleisch and Prentice (1980), Kiefer (1988) and Ridder (1990). This facilitates the inclusion and interpretation of an autoregressive dynamic structure in the model, see also Engle and Russell (1998) who consider a similar approach in modeling financial transaction data. The hazard we consider thus reads as

$$
\lambda_{i, n}\left(t \mid x_{i, n}(t), \theta_{i}\right)=\exp \left(-x_{i, n}(t)^{\prime} \beta_{i}\right) \lambda_{0}\left(t \exp \left(-x_{i, n}(t)^{\prime} \beta_{i}\right) \mid \delta_{i}\right)
$$

where $\theta_{i}=\left(\beta_{i}, \delta_{i}\right)$ and where $\lambda_{0}(u \mid \delta)$ denotes the baseline hazard. In case of the loglogistic distribution, this baseline hazard is defined as

$$
\lambda_{0}(u \mid \delta)=\frac{\delta u^{\delta-1}}{1+u^{\delta}}
$$

The hazard function then becomes

$$
\begin{aligned}
\lambda_{i, n}\left(t \mid x_{i, n}(t), \theta_{i}\right) & =\exp \left(-x_{i, n}(t)^{\prime} \beta_{i}\right) \lambda_{0}\left(t \exp \left(-x_{i, n}(t)^{\prime} \beta_{i}\right)\right) \\
& =\frac{\delta_{i} t^{\delta_{i}-1} \exp \left(-x_{i, n}(t)^{\prime} \beta_{i}\right)^{\delta_{i}}}{1+t^{\delta_{i}} \exp \left(-x_{i, n}(t)^{\prime} \beta_{i}\right)^{\delta_{i}}} .
\end{aligned}
$$


When the covariates for the $n$-th spell for household $i$ are constant over the interval $(a, b]$, the integrated baseline hazard equals

$$
\begin{aligned}
& \int_{a}^{b} \lambda_{i, n}\left(u \mid x_{i, n}(a), \theta_{i}\right) d u= \\
& \log \left[1+b^{\delta_{i}} \exp \left(-x_{i, n}(a)^{\prime} \beta_{i}\right)^{\delta_{i}}\right]-\log \left[1+a^{\delta_{i}} \exp \left(-x_{i, n}(a)^{\prime} \beta_{i}\right)^{\delta_{i}}\right] .
\end{aligned}
$$

This result can be used to compute (3). The density function for observation $t_{i, n}$ can be expressed in terms of the hazard function and the integrated hazard function, that is,

$$
f_{i, n}\left(t_{i, n} \mid x_{i, n}(t), \theta_{i}\right)=\lambda_{i, n}\left(t_{i, n} \mid x_{i, n}(t), \theta_{i}\right) S_{i, n}\left(t_{i, n} \mid x_{i, n}(t), \theta_{i}\right)
$$

where $S_{i, n}\left(t_{i, n} \mid x_{i, n}(t), \theta_{i}\right)=\exp \left(-\Lambda_{i, n}\left(t_{i, n} \mid x_{i, n}(t), \theta_{i}\right)\right)$ denotes the survivor function. Note that the density function depends on the integrated hazard function and therefore takes the complete history of the marketing instruments into account.

In Figure 2 we present an illustrative example of the accelerated failure-time hazard model with time-varying explanatory variables. In this figure the left-hand vertical axis gives the hazard rate, while the right-hand vertical axis gives the "score" $\left(x_{i, n}(t)^{\prime} \beta\right)$ which changes at calendar times $\tau_{l}$ (lower line). The smooth curve shows the baseline hazard, that is the hazard without correcting for the explanatory variables. Finally the kinked top line shows how the hazard is scaled and stretched as a result of the time-varying explanatory variables. A low "score" $x_{i, n}(t)^{\prime} \beta$ yields a higher hazard rate and therefore lowers the expected interpurchase time.

\subsection{Dynamics}

The model discussed in the previous section is static, in the sense that interpurchase times are only explained by current explanatory variables. It is however likely that the interpurchase times of households are correlated over time. For example, promotional activities may not only have an effect on current but also on future interpurchase times.

A flexible specification of these dynamic patterns is obtained by adding lagged interpurchase times and by incorporating the value of the marketing instruments at the last purchase. Technically speaking, we add $\ln t_{i, n-1}$ and $x_{i, n-1}\left(t_{i, n-1}\right)$ to explain the $n$-th 
interpurchase time of household $i$. Defining $w_{i, n}(t)=\left(x_{i, n}(t), \ln t_{i, n-1}, x_{i, n-1}\left(t_{i, n-1}\right)\right)$ and $\gamma_{i}=\left(\alpha_{i}, \rho_{i}, \omega_{i}\right)$, we can easily obtain the hazard specification for the dynamic case from (6) by replacing $x_{i, n}(t)^{\prime} \beta_{i}$ with $w_{i, n}(t)^{\prime} \gamma_{i}$.

The hazard corresponding with this dynamic duration model has to be defined as a conditional hazard given the previous interpurchase time. This conditional hazard function for $t_{i, n}$ given $t_{i, n-1}$ reads as

$$
\lambda_{i, n}\left(t \mid w_{i, n}(t), \theta_{i}\right)=\frac{\delta_{i} t^{\delta_{i}-1} \exp \left(-w_{i, n}(t)^{\prime} \gamma_{i}\right)^{\delta_{i}}}{1+t^{\delta_{i}} \exp \left(-w_{i, n}(t)^{\prime} \gamma_{i}\right)^{\delta_{i}}}
$$

with $\theta_{i}=\left(\gamma_{i}, \delta_{i}\right)$ and note that $w_{i, n}(t)$ contains $\ln t_{i, n-1}$. The density function of the timing of the $n$-th purchase occasion of household $i$ given $t_{i, n-1}$ is therefore

$$
f_{i, n}\left(t \mid w_{i, n}(t), \theta_{i}\right)=\lambda_{i, n}\left(t \mid w_{i, n}(t), \theta_{i}\right) \exp \left(-\Lambda_{i, n}\left(t \mid w_{i, n}(t), \theta_{i}\right)\right),
$$

where $\Lambda_{i, n}\left(t \mid w_{i, n}(t), \theta_{i}\right)$ is the integrated hazard function.

\subsection{Interpretation of dynamics}

For a straightforward interpretation of the parameters in the dynamic specification of the duration model, it is useful to consider the effects of a promotion during a spell. More specifically, in this section we study the dynamic effects of a promotion starting directly following a purchase made by a focal household and the promotion will end directly after the next purchase of this household. Under this strategy the marketing instruments are constant during spells. The fact that marketing instruments do not change between purchases allows for an intuitive interpretation of the parameters.

In case the covariates are constant during spells we can denote $w_{i, n}(t)=w_{i, n}$, furthermore the survivor function now simplifies to

$$
S_{i, n}\left(t \mid w_{i, n}, \theta_{i}\right)=\frac{1}{1+\left[\exp \left(-w_{i, n}^{\prime} \gamma_{i}\right) t\right]^{\delta_{i}}} .
$$

For the distribution of $t_{i, n}^{*}=\delta_{i}\left(\ln t_{i, n}-w_{i, n}^{\prime} \gamma_{i}\right)$, we derive that

$$
\begin{aligned}
\operatorname{Pr}\left[t_{i, n}^{*}<E\right] & =\operatorname{Pr}\left[\delta_{i}\left(\ln t_{i, n}-w_{i, n}^{\prime} \gamma_{i}\right)<E\right]=\operatorname{Pr}\left[t_{i, n}<\exp \left(x_{i, n}^{\prime} \beta_{i}+1 / \delta_{i} E\right)\right] \\
& =1-S\left(\exp \left(w_{i, n}^{\prime} \gamma_{i}+1 / \delta_{i} E\right)\right)=1-\frac{1}{1+\exp \left(1 / \delta_{i} E\right)^{\delta_{i}}} \\
& =1-\frac{1}{1+\exp (E)} .
\end{aligned}
$$


Hence, in the case of constant regressors, $t_{i, n}^{*}$ has a logistic density. As its density does not depend on covariates and model parameters, we can linearize the duration model as

$$
\begin{aligned}
\ln t_{i, n} & =w_{i, n}^{\prime} \gamma_{i}+\sigma_{i} \eta_{i, n} \\
& =\rho_{i} \ln t_{i, n-1}+x_{i, n}^{\prime} \alpha_{i}+x_{i, n-1}^{\prime} \omega_{i}+\sigma_{i} \eta_{i, n}
\end{aligned}
$$

where $\sigma_{i}=1 / \delta_{i}$, and where $\eta_{i, n}$ is logistic distributed such that $\mathrm{E}\left[\eta_{i, n}\right]=0$. Note that under the restriction of constant covariates, (13) is an exact alternative representation of the hazard model in (9) under constant covariates during spells.

Following ideas from the area of time-series analysis, we further rewrite (13) into the so-called error-correction format, that is,

$$
\Delta \ln t_{i, n}=\Delta x_{i, n}^{\prime} \alpha_{i}+\left(\rho_{i}-1\right)\left(\ln t_{i, n-1}-x_{i, n-1}^{\prime} \beta_{i}\right)+\sigma_{i} \eta_{i, n}
$$

where $\beta_{i}=\left(\alpha_{i}+\omega_{i}\right) /\left(1-\rho_{i}\right)$ and where $\Delta$ is the first difference operator defined as $\Delta z_{i, n}=z_{i, n}-z_{i, n-1}$, where $z_{i, n}$ can be $\ln t_{i, n}$ or $x_{i, n}$. To exclude the implausible explosive behavior of the interpurchase times, we impose that $\left|\rho_{i}\right|<1$, for all $i$. The term $\Delta x_{i, n}^{\prime} \alpha_{i}$ in (14) concerns the short-run effects of a change in $x_{i, n}$ on the interpurchase time, while the term $-x_{i, n-1}^{\prime} \beta_{i}$ in the so-called error correction part concerns the long-run effects. Notice that we cannot estimate different short- and long-run effects of variables that do not change during the time period considered, like for example household size, as then $\Delta x_{i, n}$ will be zero and $\alpha_{i}$ is not identified. We label the model in (14) the error correction model [ECM] and it will be the main specification in the rest of the paper. The long-run effects in this specification may differ in size or even in sign from short-run effects.

Following the usual time series terminology, see Hendry et al. (1984), there may be a restricted specification that is relevant. First of all, we can restrict the short-run and the long-run parameters to be equal $\left(\alpha_{i}=\beta_{i}\right)$. The resulting specification is known as the common-factor specification. Under this specification, (13) transforms into

$$
\left(\ln t_{i, n}-x_{i, n}^{\prime} \beta_{i}\right)=\rho_{i}\left(\ln t_{i, n-1}-x_{i, n-1}^{\prime} \beta_{i}\right)+\sigma_{i} \eta_{i, n}
$$

The autoregressive parameter $\rho_{i}$ is in this case equal to the correlation between $\left(\ln t_{i, n}-\right.$ $\left.x_{i, n}^{\prime} \beta_{i}\right)$ and $\left(\ln t_{i, n-1}-x_{i, n-1}^{\prime} \beta_{i}\right)$. This specification is equivalent to a model where only 
contemporaneous explanatory variables are included and where the error term follows an $\mathrm{AR}(1)$ model, that is (15) is equivalent to

$$
\begin{aligned}
\ln t_{i, n} & =w_{i, n}^{\prime} \gamma_{i}+\sigma_{i} \eta_{i, n} \\
\eta_{i, n} & =\rho_{i} \eta_{i, n-1}+u_{i, n}
\end{aligned}
$$

where $u_{i, n}$ is again an unobserved error term for which we take the same distributional assumptions as for $\eta_{i, n}$. If we additionally impose $\rho_{i}$ to be zero we obtain a static specification in which there are no dynamic effects.

\subsubsection{Effects of a promotion during spells}

We next analyze the dynamic effects of the explanatory variables on interpurchase times. The short-run effect of a marketing instrument is defined as the instantaneous effect of its (permanent) change on the interpurchase time. The long-run effect measures the effect of a permanent change of a marketing instrument at time $t^{\prime}$ on the interpurchase times at $t$ as $t \rightarrow \infty$. We focus on the error correction duration model (14) as this model nests the common factor representation (15) $\left(\alpha_{i}=\beta_{i}\right)$ and the static model $\left(\alpha_{i}=\beta_{i}\right.$ and $\left.\rho_{i}=0\right)$.

First, we consider the derivative of $\ln t_{i, n}$ with respect to $x_{i, n}$, that is,

$$
\frac{\partial \ln t_{i, n}}{\partial x_{i, n}}=\alpha_{i}
$$

Hence, an $\varepsilon$ change in $x_{i, n}$, for example due to a price reduction or a promotional activity, leads to $\alpha_{i} \varepsilon$ change in the log current interpurchase time. Note that if $x_{i, n}$ is for example the natural $\log$ of a variable, we can interpret $\alpha_{i}$ as an elasticity.

To analyze the effects of changes in the explanatory variables on future log interpurchase times, we can follow a similar procedure. The partial derivative of $\ln t_{i, n+1}$ with respect to $x_{i, n}$ is given by

$$
\frac{\partial \ln t_{i, n+1}}{\partial x_{i, n}}=-\alpha_{i}-\left(\rho_{i}-1\right) \beta_{i}+\rho_{i} \frac{\partial \ln t_{i, n}}{\partial x_{i, n}}=\left(\rho_{i}-1\right)\left(\alpha_{i}-\beta_{i}\right) .
$$

An $\varepsilon$ change in $x_{i, n}$ leads to a change of $\varepsilon\left(\rho_{i}-1\right)\left(\alpha_{i}-\beta_{i}\right)$ in $\ln t_{i, n+1}$. The derivative is zero if $\alpha_{i}=\beta_{i}$. Note that the case with $\rho_{i}=1$ is ruled out to avoid explosive interpurchase times. Hence, the common factor specification (15) (and of course the static model) 
imposes that changes in $x_{i, n}$ have no effect on the next interpurchase time. If $\beta_{i}<\alpha_{i}$, some of the effect of the change in $x_{i, n}$ on the current interpurchase time is compensated by an opposite effect on the next interpurchase time.

For the subsequent interpurchase time it holds that

$$
\frac{\partial \ln t_{i, n+2}}{\partial x_{i, n}}=\rho_{i} \frac{\partial \ln t_{i, n+1}}{\partial x_{i, n}}=\rho_{i}\left(\rho_{i}-1\right)\left(\alpha_{i}-\beta_{i}\right) .
$$

To derive the partial derivative of $\ln t_{i, n+k}$ with respect to $x_{i, n}$, we note that for $r>2$ $\partial \ln t_{i, n+r} / \partial x_{i, n}=\rho_{i} \partial \ln t_{i, n+r-1} / \partial x_{i, n}$ and hence that

$$
\frac{\partial \ln t_{i, n+k}}{\partial x_{i, n}}=\rho_{i}^{(k-1)}\left(\rho_{i}-1\right)\left(\alpha_{i}-\beta_{i}\right)
$$

If $\left|\rho_{i}\right|<1$ the effect of a change in $x_{i, n}$ on future interpurchase times will decline exponentially, and eventually it becomes zero.

From the above exercise it can already be understood that permanent changes in interpurchase times can only be obtained when $x_{i, n}$ changes permanently. For example, our model implies that only a permanent lower price can generate a permanent reduction in interpurchase times. To derive the long-run effects of a permanent change in $x_{i, n}$, we apply repeated backward substitution to (14) and obtain

$$
\begin{aligned}
\ln t_{i, n}= & \rho_{i} \ln t_{i, n-1}+\Delta x_{i, n}^{\prime} \alpha_{i}-\left(\rho_{i}-1\right) x_{i, n-1}^{\prime} \beta_{i}+\sigma_{i} \eta_{i, n} \\
= & \rho_{i}^{2} \ln t_{i, n-2}+\Delta x_{i, n}^{\prime} \alpha_{i}+\rho_{i} \Delta x_{i, n-1}^{\prime} \alpha_{i} \\
& \quad-\left(\rho_{i}-1\right) x_{i, n-1}^{\prime} \beta_{i}-\rho_{i}\left(\rho_{i}-1\right) x_{i, n-2}^{\prime} \beta_{i}+\sigma_{i} \eta_{i, n}+\rho_{i} \sigma_{i} \eta_{i, n-1} \\
= & \rho_{i}^{n} \ln t_{i, 0}+\sum_{j=0}^{n-1} \rho_{i}^{j}\left(\Delta x_{i, n-j}^{\prime} \alpha_{i}-\left(\rho_{i}-1\right) x_{i, n-j-1}^{\prime} \beta_{i}+\sigma_{i} \eta_{i, n-j}\right),
\end{aligned}
$$

where $t_{i, 0}$ denotes the pre-sample starting value of $t_{i, n}$. As $\left|\rho_{i}\right|<1, \rho_{i}^{n} \rightarrow 0$ for large $n$ and the influence of $\ln t_{i, 0}$ can be neglected. If we further impose that $x_{i, n}$ is fixed over the purchase occasions, that is $x_{i}=x_{i, n}=x_{i, n-j}, j=1, \ldots, \infty$, then for $n \rightarrow \infty,(21)$ becomes

$$
\ln t_{i, n}=\sum_{j=0}^{\infty} \rho_{i}^{j}\left(-\left(\rho_{i}-1\right) x_{i}^{\prime} \beta_{i}+\sigma_{i} \eta_{i, n-j}\right)=x_{i}^{\prime} \beta_{i}+\sum_{j=0}^{\infty} \rho_{i}^{j} \sigma_{i} \eta_{i, n-j}
$$


Hence, as $\mathrm{E}\left[\eta_{i, n-j}\right]=0$ for all $j$, the long-run expectation of $\ln t_{i, n}$ given $x_{i}$ is

$$
\mathrm{E}\left[\ln t_{i, n} \mid x_{i}\right]=x_{i}^{\prime} \beta_{i}
$$

If follows from (23) that the long-run effect of a permanent change in $x_{i}$ on the log interpurchase time is $\beta_{i}$. In sum, our error correction model for interpurchase times has short-run effects $\alpha_{i}$ and long-run effects $\beta_{i}$. Note again that in the common factor model these effects both equal $\beta_{i}$ and in the static model there are no dynamic effects at all.

Finally, to describe the dynamics in the interpurchase time model, we obtain the effects of an unexplained shock during the $n$-th interpurchase time on the subsequent purchase timings. The effect of a shock on future interpurchase times can easily be obtained from (21). The effect of an $\varepsilon$ shock during the $n$-th interpurchase spell on the $n+k$-th spell is given by $\rho_{i}^{k} \varepsilon$.

Table 1 gives an overview of all the dynamic effects for several relevant version of the model, that is the static version $\left(\rho_{i}=0, \alpha_{i}=\beta_{i}\right)$, the common factor model $\left(\rho_{i} \neq 0, \alpha_{i}=\right.$ $\left.\beta_{i}\right)$, the error correction model with $\rho_{i}=0$ and the error correction model with unrestricted $\rho_{i}$. For ease of exposition we suppress the index $i$ in this table. The table clearly shows the differences across the models. The static model does not allow for dynamic effects. The common factor model only captures the dynamics through unexplained shocks. The error correction model with $\rho_{i}=0$ does not capture dynamics in shocks but allows the effect of a marketing effort to carry over to the next interpurchase spell. Depending on the estimated difference between $\alpha_{i}$ and $\beta_{i}$, the effect on this next spell may be positive or negative. Finally, the unrestricted error correction model allows for dynamics through shocks, for multi-period carry-over effects of marketing instruments and it allows that the effect of a permanent change in a marketing instrument smoothly evolves over time.

\subsection{Parameter Estimation}

Differences in interpurchase times across households may only be partly captured by including household-specific explanatory variables in the model. Furthermore, it is also not unlikely that households may react differently to promotional activities. Therefore, 
we allow for household-specific parameters. Using similar arguments as for brand choice, neglecting this household heterogeneity may lead to an overestimate of the persistence (in our case $\rho_{i}$ ) in interpurchase times. See for example Keane (1997) for a discussion of the effects of neglecting household heterogeneity on state dependence in brand choice.

Estimation of these household-specific parameters may however be difficult if we do not have enough observations for each household. To circumvent this problem, one usually assumes that the parameters are draws from a certain population distribution. This approach is followed in the brand choice models in for example Kamakura and Russell (1989), Chintagunta et al. (1991) and Gönül and Srinivasan (1993b) among others.

A convenient choice is to assume that the parameters are draws from a finite mixture distribution which approximates the household heterogeneity distribution, see Jain et al. (1994) and Allenby and Rossi (1999) among others. The density function for household $i$ then becomes

$$
g_{i}\left(t_{i, 1}, \ldots, t_{i, N_{i}} \mid \theta\right)=\sum_{m=1}^{M} p_{m} h_{i}\left(t_{i, 1}, \ldots, t_{i, N_{i}} \mid \theta_{m}\right),
$$

where $M$ denotes the number of mixture components with $0<p_{m}<1, m=1, \ldots, M$ and $\sum_{m=1}^{M} p_{m}=1$, and where $\theta$ collects the parameters and $h\left(t_{i, 1}, \ldots, t_{i, N_{i}} \mid \theta_{m}\right)$ is the density function conditioned on segment $m$, defined as

$$
\begin{aligned}
h_{i}\left(t_{i, 1}, \ldots, t_{i, N_{i}} \mid \theta_{m}\right) & = \\
& f_{i, 1}\left(t_{i, 1} \mid x_{i, 1}, \theta_{m}\right) S_{i, N_{i}}\left(t_{i, N_{i}} \mid w_{i, N_{i}}(t), \theta_{m}\right) \prod_{n=2}^{N_{i}-1} f_{i, n}\left(t_{i, n} \mid w_{i, n}(t), \theta_{m}\right),
\end{aligned}
$$

where the density function $f_{i, n}\left(t_{i, n} \mid w_{i, n}(t), \theta_{m}\right)$ is given in (10). The second term, involving the survivor function, is included for the last observation of household $i$ when it is censored from the right, see for example Kiefer (1988) for a discussion. If there is no censoring, one can simply remove this term and replace the upper limit of the sum by $N_{i}$.

The density for the first observation is denoted by $f_{i, 1}\left(t_{i, 1} \mid x_{i, 1}, \theta_{m}\right)$. For the first interpurchase time we do not observe the lagged interpurchase time. Instead of fixing its value, we choose to describe the initial observation by the long-run relation between interpurchase times and the marketing instruments in (23). To be more specific, we take 
the initial observation as

$$
\ln t_{i, 1}=\mu_{0, i}+x_{i, 1}^{\prime} \beta_{i}+\tilde{\sigma}_{i} \eta_{i, 1}
$$

where $\eta_{i, 1}$ has a logistic distribution. Note that we allow for a different intercept and scale parameter for the initial observation for flexibility reasons.

The parameters of duration models generally can be estimated using maximum likelihood [ML]. The log likelihood function is given by

$$
\ell(\theta)=\sum_{i=1}^{I} \ln \left(g_{i}\left(t_{i, 1}, \ldots, t_{i, N_{i}} \mid \theta\right)\right),
$$

where $g_{i}\left(t_{i, 1}, \ldots, t_{i, N_{i}} \mid \theta\right)$ is defined in (24). This log likelihood function can be maximized using standard numerical optimization algorithms. In case of household heterogeneity one may opt for the EM-algorithm of Dempster et al. (1977). The resulting maximum likelihood estimator denoted by $\hat{\theta}$ is normally distributed with mean $\theta$ and the information matrix as covariance matrix. To compute this covariance matrix, we take the outer product of gradients.

Parameter estimates for the static duration model and the common factor duration model (15) can be obtained in a similar way. As both models are nested in the error correction model (14), we can use standard likelihood ratio tests to compare the three models. For instance, under the parameter restriction $\alpha_{m}=\beta_{m}$ for $m=1, \ldots, M$ the error correction duration model (14) simplifies to the common factor model (15). To compare both models, we can perform a likelihood ratio test for the hypothesis $\alpha_{m}=\beta_{m}$. The corresponding likelihood ratio test statistic, is asymptotically $\chi^{2}(J)$ distributed under the null hypothesis, where $J$ denotes the number of parameter restrictions.

It should be stressed that the likelihood ratio test procedure to compare two model specifications is only valid if the two models under consideration are nested. They should then have the same number of mixture components $M$ to describe household heterogeneity. If the number of mixture components is different in the two model specifications, the test includes a test for the number of mixture components $M$. Likelihood ratio tests for the number of mixture components $M$ are not asymptotically $\chi^{2}$-distributed. To illustrate this, consider a common factor model with two mixture components $(M=2)$. Under 
the restriction $\beta_{1}=\beta_{2}$ the mixing proportion $p_{1}$ is not identified and the likelihood ratio test statistic for $\beta_{1}=\beta_{2}$ is not asymptotically $\chi^{2}$-distributed under the null hypothesis. This phenomenon is known as the Davies (1977) problem. We will abstain from a further analysis of this issue here, and in our empirical work we will use the out-of-sample loglikelihood to determine the value of $M$.

\section{Application}

In this section we illustrate the dynamic duration models on scanner panel data on purchases of fast-moving consumer goods in three different categories. In Section 3.1, we discuss the data. In Section 3.2, we consider the maximum likelihood estimates of various duration models and we examine the presence of dynamic effects in interpurchase times. In Section 3.3, we use the estimation results to analyze the short-run and long-run effects of promotions on interpurchase times.

\subsection{The Data}

The data we use are A.C. Nielsen household scanner panel data on purchases in three different categories from 1985 to 1988 in Sioux Falls, South Dakota. The three categories are liquid laundry detergent, catsup and yogurt. These three categories differ substantially in their average purchase rate, consumption patterns and storability. The dynamic patterns in the purchase timing are likely to differ across these categories. A subset of these data are analyzed in Chintagunta and Prasad (1998) using a Dynamic McFadden Model. We aggregate marketing efforts to a weekly level as, in fast moving consumer goods markets, these efforts tend to be constant during a week, where the week is defined from Wednesday to Tuesday. We do allow households to have multiple purchase occasions during one week.

For each category we select households buying only of the top brands. The top brands are defined as those brands that are sold frequently enough to build up the entire marketing effort history. This selection will delete more households in some categories than in others. Furthermore, we select households which are observed to buy at least four times 
in the observational period. Table 2 shows an overview of the data. This table shows that, compared to the catsup category, there are less households for the detergent and the yogurt categories. The main reason seems to be that there are just a few (main) brands in the catsup category. For the other two categories there are more main brands but also many smaller brands. Households buying such small brands have to be completely removed from the data. The selected brands account for almost $90 \%$ of the market in all three categories. However, the selected households, that is, those never buying another brand, only account for 38, 35 and 59\% for yogurt, detergent and catsup, respectively. Furthermore, we trim the number of weeks to yield a period in which all brands are available. For example, for the detergent category the data contain a brand introduction and for this category we start our analysis after this event.

For each purchase occasion, we know the timing and the volume purchased. Furthermore, for each week we know the shelf price (dollars/32oz.) of all brands and which brands are featured or displayed. As the interpurchase time is defined at the category level we need to aggregate the marketing information over stores and brands. To keep as much information as possible, we use household-specific weights in this aggregation. Following Gupta (1991), we use household-specific volume brand shares to aggregate over brands. Aggregation over stores is done using household-specific store weights. Note that, by using this weighting scheme, we use for each household only data on the relevant store and brand options. The easier method, that is, using the marketing instruments of the store actually visited and the brand actually chosen, is not an option here. This is because in the weeks between purchases, we have not yet observed the brand choice and therefore this information cannot be used. Due to this aggregation, the display and feature variables represent the percentage of stores featuring a brand, or having the brand on display in the category. Next to information on marketing activities and purchase timing, we also have access to some household characteristics. In our purchase timing models we use the household size, household income and the volume purchased at the previous purchase occasion.

Tables 2 and 3 give some summary statistics on the three categories and the ex- 
planatory variables. After the above-mentioned selections, we are left with at least 585 households in each category and over 7000 observed interpurchase spells. We have the most data for the catsup category, that is 1435 households with 14489 interpurchase spells. The mean interpurchase time ranges from 5 to 10 weeks. Note that marketing instruments in Table 3 are averaged over weeks, stores and different UPCs. The display and feature variables therefore take values between 0 and 1 . The average levels of these variables seem quite small, but there are many weeks in which some UPCs were on display or featured.

\subsection{Estimation Results}

To analyze interpurchase times, we consider three versions of our model, that is, the commonly considered static duration model, the common factor duration model (15), and our error correction duration model (14), where this last model is the most flexible. Note that we do not intend to model brand choice and purchase quantity, at least, not in this paper. As explanatory variables we use household size, household income and the volume purchased at the previous purchase occasion (divided by 32 oz.). The latter variable is used as a proxy for "regular" and "fill-in" trips and it also accounts for the effects of household inventory behavior on purchase timing, see also Chintagunta and Prasad (1998). Furthermore, we use the actual price in dollars per 32 oz. Finally, two variables are used to indicate whether brands were on display or were featured.

To select the optimal model for each category, we use the following model selection strategy. First of all, we select the optimal number of segments to use to capture the heterogeneity. To this end, we estimate the error correction specification (14) of the duration model for different numbers of segments. The performance of each model is measured using the log likelihood on an out-of-sample selection of households. We use $75 \%$ of the households for parameter estimation and the remaining $25 \%$ are the outof-sample households. The number of segments yielding the highest out-of-sample log likelihood is then selected. Note that by using an out-of-sample measure to select the number of segments, we reduce the probability of overfitting the data. For the selected number of segments, we test whether we can restrict the dynamic structure of the error 
correction model to a common factor (15) or to a static representation. As these last two models are nested within the error correction specification, we can use Likelihood Ratio $[\mathrm{LR}]$ tests to test for restricted dynamic structures.

For the catsup and the yogurt category, it turns out that four segments are sufficient to capture the heterogeneity, while for the detergent category we need six segments. Upon using LR-tests, the static and the common-factor specification are rejected against the error-correction model for all categories. This shows that there are indeed significant dynamic effects in interpurchase timing. Furthermore, as the common-factor model is rejected against the error-correction model, short-run effects apparently differ from the corresponding long-run effects.

In Table 4 and Table 5, we present the estimation results for the three categories for the final models. Table 4 shows the mean parameters over the sample, that is $\sum_{m=1}^{M} \hat{p}_{m} \hat{\theta}_{m}$. Parameters in boldface are significant at 5\%. In parentheses, the table gives the segment numbers for which the (segment-specific) parameters are significantly different from zero. Note that the segments are ordered such that segment 1 is the largest. Next, Table 5 shows the average parameter estimates over the segments for which there is a significant effect. This table also gives the corresponding fraction of the sample. This table will be especially useful to compare the three categories.

Tables 4 and 5 display the parameter estimates of the error correction duration model (14) for the yogurt category in the second column. As household size and income are constant over the time period considered, we cannot estimate a different short-run and long-run effect of these variables. Price has a significant short-run effect for the first two segments $(88.5 \%)$ of the sample and a significant long-run effect for the first and the third segment (78.5\%). Display only has a significant short-run effect for segment 1. Finally, the $\rho$ parameter is significant for all but the smallest segment of the sample, its mean equals 0.199 . Therefore, on average, $20 \%$ of a shock to the interpurchase time is carried over to the next spell.

The third column shows the estimation results for the detergent category. Display has significant short-run and long-run effects for $76.7 \%$ and $43.2 \%$ of the sample, respectively, 
where now the short-run effect is larger. In comparison to the yogurt category, the purchased volume has a much larger effect on the purchase timing of detergent. If a household buys a large quantity of detergent, it is likely that the next observed interpurchase time will be longer than on average. An obvious explanation is that this product can be easily held on stock, in contrast to yogurt. For yogurt, one may expect the usage rate to increase with the purchased quantity. In the detergent category, the autocorrelation parameter $\rho$ is only significant for the smallest categories. Averaged over these two categories the estimate of $\rho$ equals 0.231. Although on average there is no significant correlation, for $9.5 \%$ of the sample we do find a significant autoregressive relation.

The third category we study concerns catsup. For this category we do not find a significant $\rho$-parameter for any segment. The speed of convergence to the steady state for this category is therefore very large. Not surprisingly, this category has the largest mean interpurchase time, see Table 2. Purchase occasions tend to be relatively far apart in (calendar) time, leading to a smaller correlation between interpurchase times. We however do find significantly different long-run and short-run effects. Surprisingly, for the largest segment of households a price increase decreases interpurchase times on the long-run. A possible explanation for this finding is that households may buy smaller quantities/sizes of catsup when the price is high. A permanent increase in price will in that case lead to smaller interpurchase times. Finally, we see that previous purchases only have a short-run effect, thereby again illustrating that an error-correction model can yield useful inference.

When we compare the results for the three categories in Table 5, we find that (i) categories with large interpurchase times have smaller autocorrelations, that (ii) shortrun effects of marketing instruments differ significantly from the long-run effects, and that (iii) price has most long-run effect on the perishable product.

\subsection{Short-Run Effects of Promotions}

In this section we examine the short and long-run effects of specific promotion scenarios on interpurchase timing. We discuss two different scenarios, one that is not very realistic and 
one that is realistic but more difficult to evaluate. It will turn out that the first scenario does provide very useful insights in the dynamics, and hence has important managerial implications.

First of all we analyze the effects of a promotion targeted at one specific household, the promotion starts right after an observed purchase and ends at the time of the next purchase. Under this marketing plan, the marketing efforts are constant during the interpurchase spells of this household. We can therefore use the results of Section 2.3 to derive the effects of such a promotion on the future interpurchase times. Using the same results we can evaluate the effects of a permanent promotion on the purchase timing of this household.

Figure 3 shows the effects of a price promotion and the effects of a display on the interpurchase times in the yogurt category. The results are based on the parameter estimates for the largest segment in the error-correction specification (14), see the second column of Table 4. The top graph in Figure 3 shows the effects of a price cut on five consecutive interpurchase spells, where the effect is represented by the partial derivative of the log interpurchase time to the marketing instrument. We see that a temporary price cut (solid line) has a strong negative effect on the first interpurchase time. The effect on the next interpurchase times is negative as well, but the size of the effect quickly converges to zero. Only for the first two interpurchase times does the price cut have a substantive impact. As the carry-over effects have the same sign as the direct effect, the long-run effect of a permanent price cut (dashed line) is larger than the direct effect.

For display, we obtain a different pattern. Although display shortens the first interpurchase time the next ones are expected to be larger than normal. For display, it therefore holds that the long-run effect of a permanent display is smaller than the direct effect. Actually the long-run effect for display is not significantly different from zero for any segment, see Table 4. Display therefore mainly has short-run effects, while price has short as well as long-run effects.

The above analysis is directly obtained from the parameter estimates. However, it only gives the effects for a very specific situation, that is, the effects of a promotion targeted 
at a single household. In practice one is interested in the effects on a more aggregate level and the promotion will then not start at the beginning of the interpurchase spells of all households. A more realistic setting is a promotion during one week. To assess the impact of such a promotion, we have to rely on simulation. We use the estimated model to simulate purchases for a number of households starting at calendar time $t_{0}$, where we have a promotion from $t_{1}$ to $t_{2}$, and for $t<t_{1}$ and $t>t_{2}$, we set the marketing instruments to their sample mean, see Table 3. The size of the promotion is set to one standard deviation. For every week, we calculate the percentage of households that would have made a purchase. The average interpurchase time for this week is the reciprocal of this percentage. Note that we now measure the absolute effects of promotion instead of marginal effects as in the previous exercise.

Figure 4 shows the effects of a promotion during one week, again for the largest segment of households in the yogurt category. Contrary to the previous analysis the current scenario analysis is done in calendar time. Interestingly, this graph shows the same general pattern as the analysis on the household level, see Figure 3, thereby demonstrating how one can use the modeling results to evaluate the effects of managerial decisions.

\section{Conclusion}

In this paper we proposed a dynamic model for interpurchase times, in which we can disentangle short-run from long-run effects of marketing variables. We discussed representation, interpretation and estimation issues. We illustrated our model for purchases on three different categories and we found that the short-run effects of marketing-mix variables can be significantly different from the long-run effects. Also, we found that the effects of marketing instruments, both in the short-run and the long-run, can vary substantially across categories. Additionally, we showed that our model can be used to evaluate marketing strategies.

A topic of further research amounts to building on the work of Gupta (1988), Chintagunta (1993), Ailawadi and Neslin (1998) and Bucklin et al. (1998), where interpurchase times or purchase incidence decisions are combined with brand choice and purchase quan- 
tity. Indeed, one could construct models for long-run and short-run effects of marketing mix variables for all marketing performance measures jointly. In this case, we could use the approach of Paap and Franses (2000) to capture dynamics in brand choice and the ideas in Böckenholt (1998) to model dynamics in purchased quantity. 


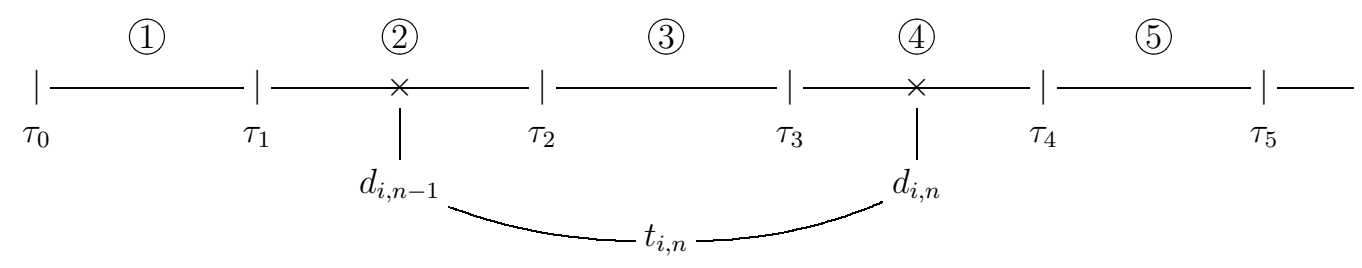

Figure 1: Graphical representation of purchase occasion $d_{i, n}$, interpurchase time $t_{i, n}$ and time indexes of changes in covariates $\tau_{l}$

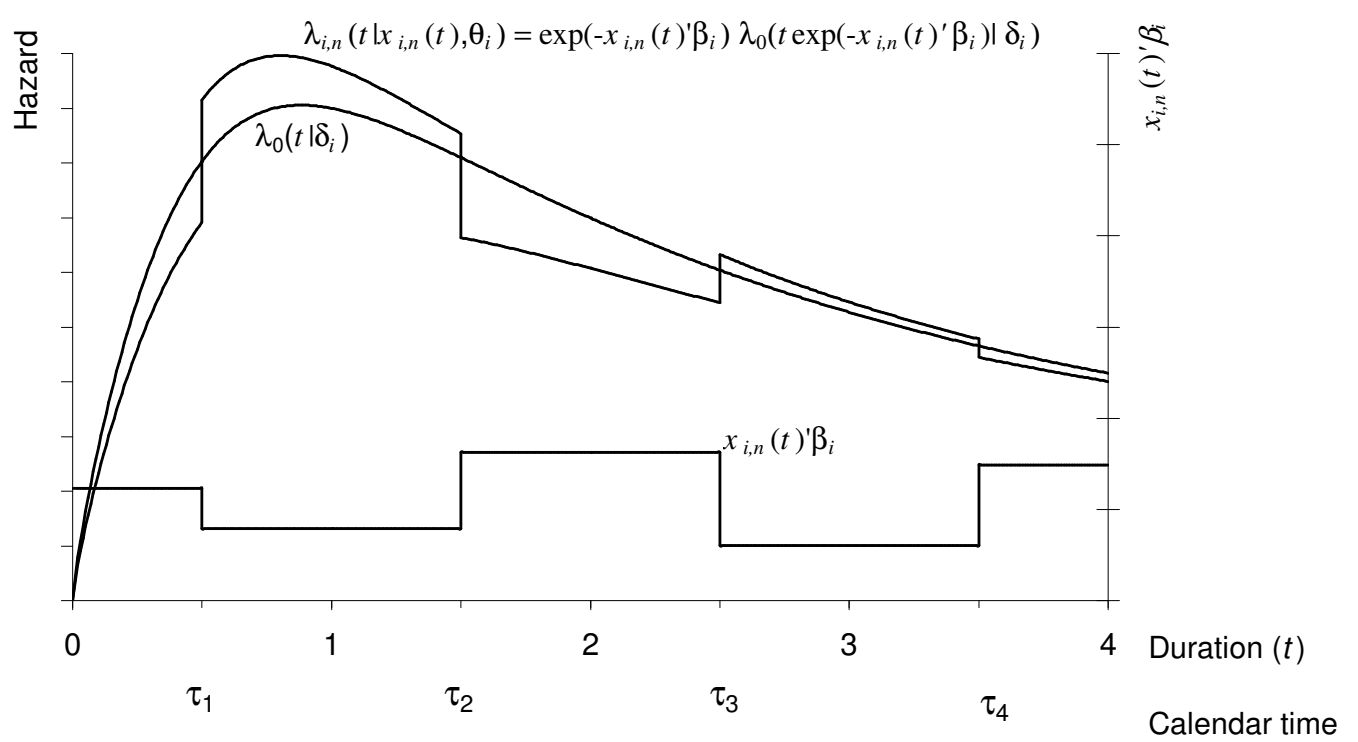

Figure 2: Illustrative example of the hazard rate for an accelerate failure-time model with time-varying explanatory variables. 


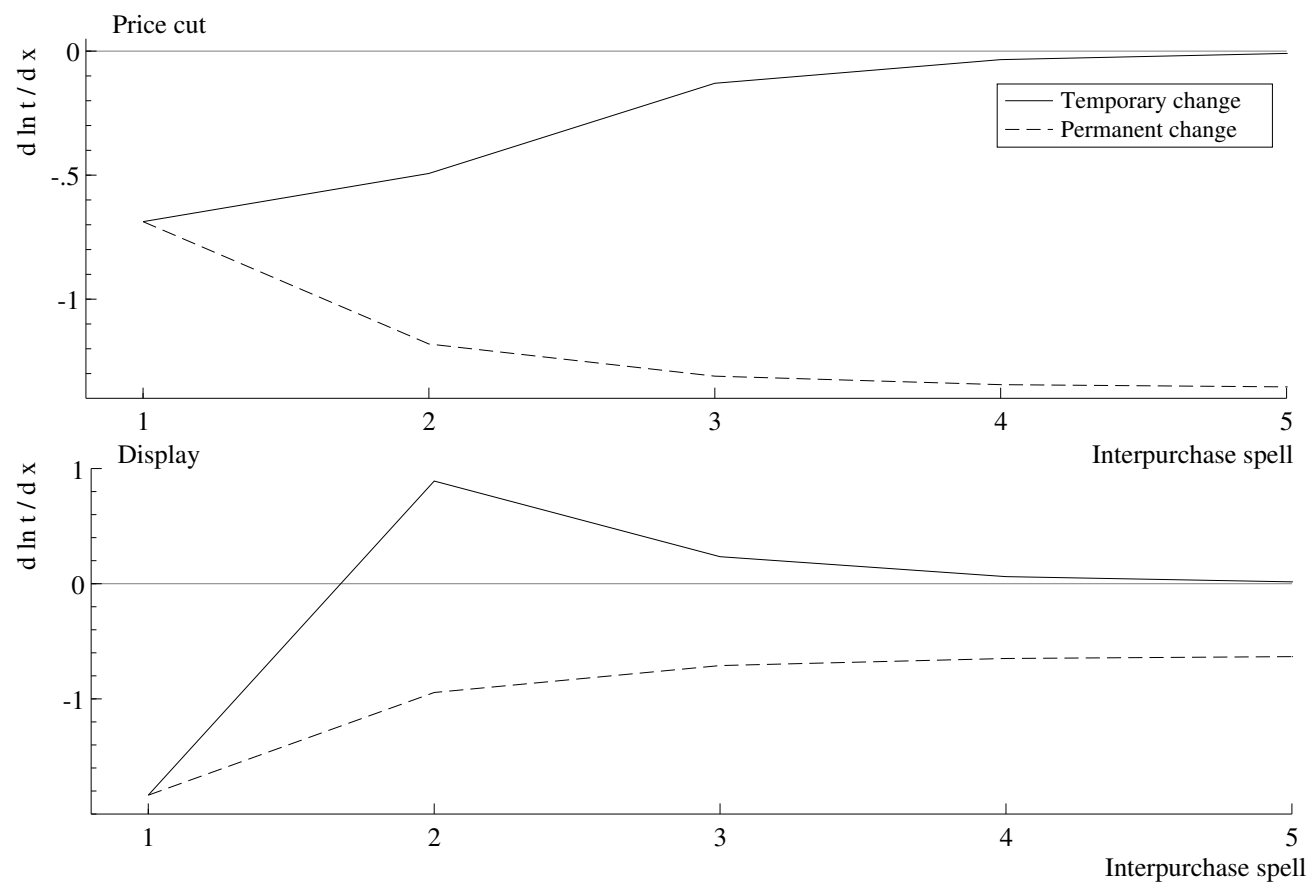

Figure 3: Graphical presentation of the effect of a temporary/permanent price cut or display on interpurchase times. Results apply to the largest segment of the four-segment error-correction specification for the yogurt category.

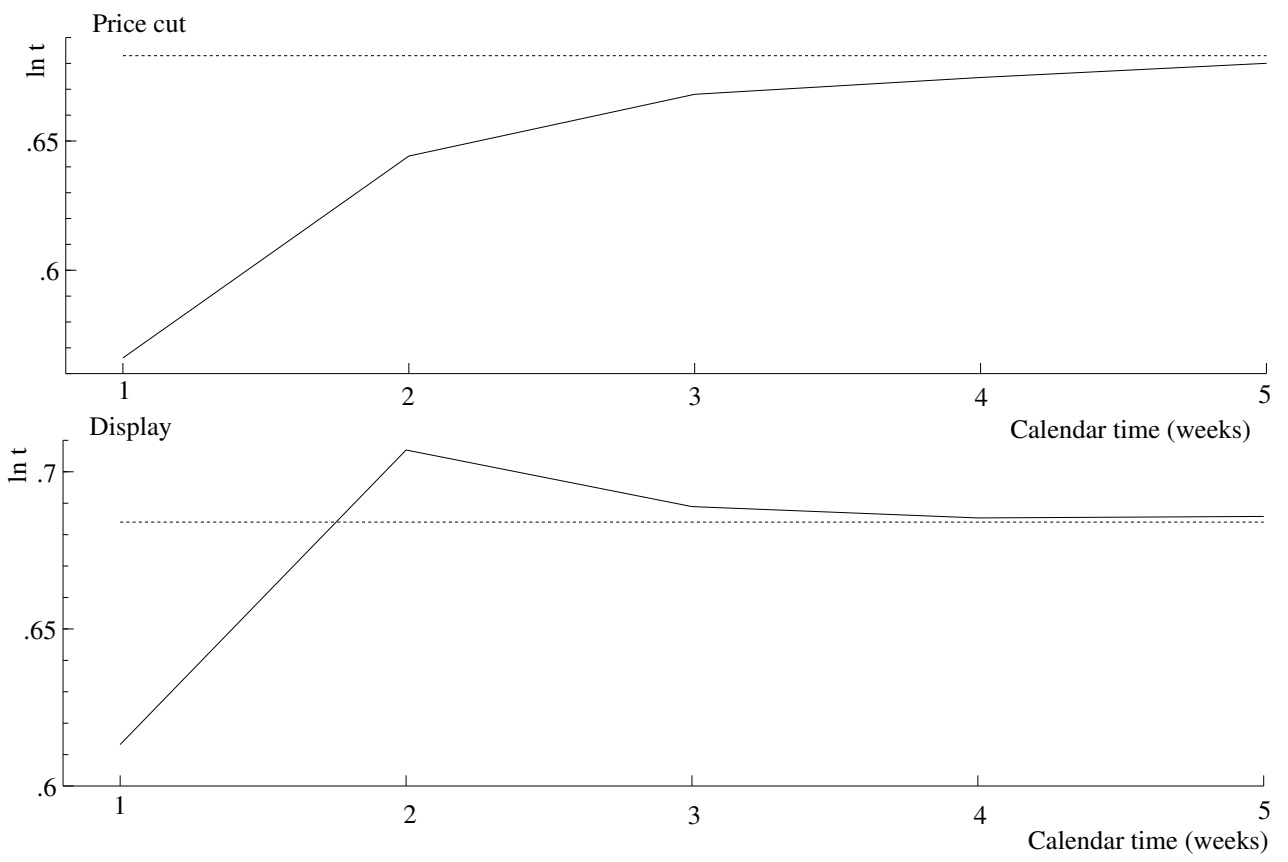

Figure 4: Graphical presentation of the effect of a promotion during one week on the average $(\log )$ interpurchase time. 
Table 1: Dynamic effects of temporary and permanent changes in marketing instruments and of unexplained shocks in different model versions

Static Common Factor $\operatorname{ECM}(\rho=0) \quad$ ECM $(0<|\rho|<1)$

\begin{tabular}{|c|c|c|c|c|}
\hline \multicolumn{5}{|c|}{ Temporary change in $x$ at time $n$} \\
\hline$\partial \ln t_{n} / \partial x_{n}$ & $\beta$ & $\beta$ & $\alpha$ & $\alpha$ \\
\hline$\partial \ln t_{n+1} / \partial x_{n}$ & 0 & 0 & $-(\alpha-\beta)$ & $(\rho-1)(\alpha-\beta)$ \\
\hline$\vdots$ & $\vdots$ & $\vdots$ & $\vdots$ & $\vdots$ \\
\hline$\partial \ln t_{n+k} / \partial x_{n}$ & 0 & 0 & 0 & $\rho^{k-1}(\rho-1)(\alpha-\beta)$ \\
\hline$\vdots$ & $\vdots$ & $\vdots$ & $\vdots$ & $\vdots$ \\
\hline$\partial \ln t_{\infty} / \partial x_{n}$ & 0 & 0 & 0 & 0 \\
\hline \multicolumn{5}{|c|}{ Permanent change in $x$ starting at time $n$} \\
\hline$\partial \ln t_{n} / \partial x$ & $\beta$ & $\beta$ & $\alpha$ & $\alpha$ \\
\hline$\partial \ln t_{n+1} / \partial x$ & $\beta$ & $\beta$ & $\beta$ & $\rho \alpha+(1-\rho) \beta$ \\
\hline$\vdots$ & $\vdots$ & $\vdots$ & $\vdots$ & $\vdots$ \\
\hline$\partial \ln t_{n+k} / \partial x$ & $\beta$ & $\beta$ & $\beta$ & $\rho^{k} \alpha+(1-\rho) \beta \sum_{i=0}^{k-1} \rho^{i}$ \\
\hline$\vdots$ & $\vdots$ & $\vdots$ & $\vdots$ & $\vdots$ \\
\hline$\partial \ln t_{\infty} / \partial x$ & $\beta$ & $\beta$ & $\beta$ & $\beta$ \\
\hline \multicolumn{5}{|c|}{ Effect of an $\varepsilon$ shock at time $n$} \\
\hline $\ln t_{n}$ & $\varepsilon$ & $\varepsilon$ & $\varepsilon$ & $\varepsilon$ \\
\hline $\ln t_{n+1}$ & 0 & $\rho \varepsilon$ & 0 & $\rho \varepsilon$ \\
\hline$\vdots$ & $\vdots$ & $\vdots$ & $\vdots$ & $\vdots$ \\
\hline $\ln t_{n+k}$ & 0 & $\rho^{k} \varepsilon$ & 0 & $\rho^{k} \varepsilon$ \\
\hline$\vdots$ & $\vdots$ & $\vdots$ & $\vdots$ & $\vdots$ \\
\hline $\ln t_{\infty}$ & 0 & 0 & 0 & 0 \\
\hline
\end{tabular}


Table 2: Data characteristics three scanner panel data sets (interpurchase time measured in weeks)

\begin{tabular}{|c|c|c|c|c|c|c|}
\hline & \# brands & \# stores & $\mathrm{T}^{\mathrm{a}}$ & $\mathrm{I}^{\mathrm{b}}$ & $\begin{array}{l}\text { Interpr } \\
\text { count }\end{array}$ & $\begin{array}{l}\text { lase time } \\
\text { mean }\end{array}$ \\
\hline Catsup & 3 & 15 & 139 & 1435 & 14489 & 10.00 \\
\hline Detergent & 13 & 13 & 97 & 624 & 7290 & 6.94 \\
\hline Yogurt & 6 & 13 & 91 & 585 & 7019 & 4.98 \\
\hline
\end{tabular}

a Number of interpurchase spells

b Number of households

Table 3: Explanatory variables

\begin{tabular}{lcccccc}
\hline & \multicolumn{3}{c}{ Brand characteristics } & \multicolumn{3}{c}{ Household char. } \\
\multicolumn{2}{c}{ Price $(\$ / 32$ oz.) } & Display (\%) & Feature (\%) & Income & Size & Volume (32 oz.) \\
\hline Catsup & 1.20 & 1.52 & 2.96 & 6.40 & 3.44 & 1.11 \\
Detergent & 1.60 & 0.60 & 0.47 & 6.00 & 3.01 & 2.77 \\
Yogurt & 2.32 & 0.88 & 2.59 & 6.16 & 2.86 & 0.76 \\
& & & & & & \\
\hline
\end{tabular}


Table 4: Sample averaged ML parameter estimates for the yogurt, catsup and detergent categories for the error-correction duration model, with household heterogeneity. Significant estimates (at 5-\% level) are given in boldface, and in parentheses we indicate the segments for which the segment-level estimate is significant.

$$
\text { yogurt detergent catsup }
$$

\begin{tabular}{|c|c|c|c|c|}
\hline \multirow[b]{2}{*}{ price (32 oz.) } & \multicolumn{4}{|c|}{ short-run parameters $(\alpha)$} \\
\hline & 0.629 & $(12)$ & 0.795 ( $2 \quad)$ & $\mathbf{1 . 1 1 5}(12 \quad)$ \\
\hline display & -1.265 & $\left(\begin{array}{ll}1 & )\end{array}\right)$ & $-\mathbf{3 . 0 1 0}(124 \quad)$ & $-\mathbf{2 . 3 4 7}$ \\
\hline feature & -0.619 & & $-0.920 \quad(24)$ & $\mathbf{- 1 . 6 5 2}$ \\
\hline \multirow[t]{2}{*}{ volume prev. (32 oz.) } & -0.020 & $(3)$ & $\mathbf{0 . 1 3 5}(12456)$ & $0.090(234)$ \\
\hline & \multicolumn{4}{|c|}{ long-run parameters $(\beta)$} \\
\hline price (32 oz.) & 1.044 & $\left(\begin{array}{ll}1 & 3\end{array}\right)$ & $0.234 \quad(2 \quad)$ & $-0.437(1 \quad)$ \\
\hline display & -0.396 & & -2.090 & $\mathbf{- 1 . 6 1 5}(12)$ \\
\hline feature & -0.045 & $(\quad)$ & $-0.963 \quad(2)$ & $-\mathbf{2 . 0 0 9}$ \\
\hline household income & -0.004 & $(\quad)$ & $-\mathbf{0 . 0 1 9}(\quad 5)$ & $0.006 \quad(2)$ \\
\hline household size & -0.040 & $(1 \quad)$ & $-\mathbf{0 . 1 7 6}$ & -0.188 \\
\hline volume prev. (32 oz.) & -0.085 & & $\mathbf{0 . 1 3 3}(12346)$ & 0.065 \\
\hline$\mu_{1}$ & 1.609 & $(123)$ & 2.148 & 2.892 \\
\hline$\mu_{0}-\mu_{1}$ & 0.067 & $(3)$ & $0.037 \quad\left(\begin{array}{ll}3 & )\end{array}\right)$ & $-0.018 \quad(\quad)$ \\
\hline$\delta_{0}$ & 1.637 & $(123)$ & $\mathbf{2 . 3 4 1}(123456)$ & 2.277 \\
\hline$\delta_{1}$ & 1.732 & $(123)$ & 2.613 (123456) & 2.008 \\
\hline$\rho$ & 0.199 & $(123)$ & $0.003 \quad(\quad 56)$ & $0.010 \quad(\quad)$ \\
\hline$p_{1}$ & \multicolumn{2}{|c|}{0.703} & 0.432 & 0.487 \\
\hline$p_{2}$ & \multicolumn{2}{|c|}{0.182} & 0.236 & 0.258 \\
\hline$p_{3}$ & \multicolumn{2}{|c|}{0.082} & 0.139 & 0.209 \\
\hline$p_{4}$ & \multicolumn{2}{|c|}{0.034} & 0.099 & 0.046 \\
\hline$p_{5}$ & \multicolumn{2}{|c|}{-} & 0.048 & - \\
\hline$p_{6}$ & \multicolumn{2}{|l|}{-} & 0.047 & - \\
\hline In-sample $\ell(\hat{\theta})$ & \multicolumn{2}{|c|}{-12927.33} & -14968.93 & -35693.80 \\
\hline \multicolumn{5}{|l|}{ LR-tests ( $p$-values) } \\
\hline static vs ECM & \multicolumn{2}{|c|}{0.000} & 0.000 & 0.000 \\
\hline common factor vs ECM & \multicolumn{2}{|c|}{0.000} & 0.000 & 0.000 \\
\hline
\end{tabular}


Table 5: Average parameter estimates over segments for which the segmentspecific estimates are significant at $5 \%$, with the corresponding fraction of the sample in parentheses.

\begin{tabular}{|c|c|c|c|c|c|c|}
\hline \multirow{6}{*}{$\begin{array}{l}\text { price }(32 \text { oz. }) \\
\text { display } \\
\text { feature } \\
\text { volume prev. ( } 32 \text { oz.) }\end{array}$} & \multicolumn{2}{|c|}{ yogurt } & \multicolumn{2}{|c|}{ detergent } & \multicolumn{2}{|c|}{ catsup } \\
\hline & \multicolumn{6}{|c|}{ short-run effects ( $\alpha$ ) } \\
\hline & 0.648 & $(88.5)$ & 1.538 & $(43.2)$ & 1.356 & $(74.5)$ \\
\hline & -1.836 & $(70.3)$ & -3.174 & $(76.7)$ & -2.347 & $(100)$ \\
\hline & - & $(0.0)$ & -2.267 & $(33.5)$ & -1.652 & $(100)$ \\
\hline & 0.414 & $(8.2)$ & 0.155 & $(86.1)$ & 0.232 & $(51.3)$ \\
\hline & \multicolumn{6}{|c|}{ long-run effects $(\beta)$} \\
\hline price (32 oz.) & 1.369 & $(78.5)$ & 0.937 & $(23.6)$ & -1.014 & $(48.7)$ \\
\hline display & - & $(0.0)$ & -4.569 & $(43.2)$ & -1.933 & $(74.5)$ \\
\hline feature & - & $(0.0)$ & -3.583 & $(23.6)$ & -2.009 & $(100)$ \\
\hline household income & - & $(0.0)$ & -0.177 & $(4.8)$ & 0.025 & $(25.8)$ \\
\hline household size & -0.056 & $(70.3)$ & -0.176 & $(100)$ & -0.188 & $(100)$ \\
\hline volume prev. (32 oz.) & - & $(0.0)$ & 0.138 & $(92.5)$ & 0.065 & $(100)$ \\
\hline$\mu_{1}$ & 1.664 & $(96.6)$ & 2.148 & $(100)$ & 2.892 & $(100)$ \\
\hline$\mu_{0}-\mu_{1}$ & 0.783 & $(8.2)$ & 0.298 & (13.9) & - & $(0.0)$ \\
\hline$\delta_{0}$ & 1.562 & $(96.6)$ & 2.341 & $(100)$ & 2.277 & $(100)$ \\
\hline$\delta_{1}$ & 1.651 & $(96.6)$ & 2.613 & $(100)$ & 2.008 & $(100)$ \\
\hline$\rho$ & 0.205 & $(96.6)$ & 0.231 & $(9.5)$ & - & $(0.0)$ \\
\hline
\end{tabular}




\section{References}

Ailawadi, K. L. and Neslin, S. A.: 1998, The effect of promotion on consumption: Buying more and consuming it faster, Journal of Marketing Research 35(3), 390-398.

Allenby, G. M., Leone, R. P. and Jen, L.: 1999, A dynamic model of purchase timing with application to direct marketing, Journal of the American Statistical Association 94(446), 365-374.

Allenby, G. M. and Rossi, P. E.: 1999, Marketing models of consumer heterogeneity, Journal of Econometrics 89, 57-78.

Blattberg, R. C., Eppen, G. C. and Liebermann, J.: 1981, A theoretical and empirical evaluation of price deals for consumer durables, Journal of Marketing 45, 116-129.

Böckenholt, U.: 1998, Mixed INAR(1) poisson regression models: Analyzing heterogeneity and serial dependencies in longitudinal count data, Journal of Econometrics 89(12), 317-338.

Bucklin, R. E., Gupta, S. and S., S.: 1998, Determining segmentation in sales response across consumer purchase behaviors, Journal of Marketing Research 35(May), 189-197.

Chintagunta, P.: 1993, Investigating purchase incidence, brand choice and purchase quantity decisions of households, Marketing Science 12(2), 184-208.

Chintagunta, P. K. and Haldar, S.: 1998, Investigating purchase timing behavior in two related product categories, Journal of Marketing Research 35, 43-53.

Chintagunta, P. K., Jain, D. C. and Vilcassim, N. J.: 1991, Investigating heterogeneity in brand preferences in logit models for panel data, Journal of Marketing Research 28, 417-428.

Chintagunta, P. K. and Prasad, A. R.: 1998, An empirical investigation of the "dynamic McFadden" model of purchase timing and brand choice: Implications for market structure, Journal of Business $\&$ Economic Statistics 16(1), 2-12. 
Davies, R. B.: 1977, Hypothesis testing when a nuisance parameter is present only under the alternative, Biometrika 64(2), 247-254.

Dekimpe, M. G., Hanssens, D. M. and Silva-Risso, J. M.: 1999, Long-run effects of price promotions in scanner markets, Journal of Econometrics 89, 269-291.

Dempster, A. P., Laird, N. M. and Rubin, D. B.: 1977, Maximum likelihood from incomplete data via the EM algorithm (with discussion), Journal of the Royal Statistical Society B 39, 1-38.

Doornik, J. A.: 1999, Object-Oriented Matrix Programming Using Ox, 3rd edn, London: Timberlake Consultants Press and Oxford: www.nuff.ox.ac.uk/Users/Doornik.

Engle, R. F. and Russell, J. R.: 1998, Autoregressive conditional duration: A new model for irregularly spaced transaction data, Econometrica 66(5), 1127-1162.

Gönül, F. and Srinivasan, K.: 1993a, Consumer purchase behavior in a frequently bought product category: Estimation issues and managerial insights from a hazard function model with heterogeneity, Journal of the American Statistical Association 88(424), 1219-1227.

Gönül, F. and Srinivasan, K.: 1993b, Modeling multiple sources of heterogeneity in multinomial logit models: Methodological and managerial issues, Marketing Science 12(3), 213-229.

Gupta, S.: 1988, Impact of sales promotions on when, what, and how much to buy, Journal of Marketing Research 25, 342-355.

Gupta, S.: 1991, Stochastic models of interpurchase time with time-dependent covariates, Journal of Marketing Research 28, 1-15.

Helsen, K. and Schmittlein, D. C.: 1992, How does a product market's typical pricepromotion pattern affect the timing of households' purchases? an empirical study using upc scanner data, Journal of Retailing 68(3), 316-338. 
Helsen, K. and Schmittlein, D. C.: 1993, Analyzing duration times in marketing: Evidence for the effectiveness of hazard rate models, Marketing Science 11(4), 395-414.

Hendry, D. F., Pagan, A. R. and Sargan, J. D.: 1984, Dynamic specification, in Z. Griliches and M. Intriligator (eds), Handbook of Econometrics, Vol. 2, North-Holland, Amsterdam, chapter 18, pp. 1023-1100.

Jain, D. C. and Vilcassim, N. J.: 1991, Investigating household purchase timing decisions: A conditional hazard function approach, Marketing Science 10(1), 1-23.

Jain, D. C., Vilcassim, N. J. and Chintagunta, P. K.: 1994, A random-coefficients logit brand-choice model applied to panel data, Journal of Business 6 Economic Statistics $\mathbf{1 2}(3), 317-328$.

Jedidi, K., Mela, C. F. and Gupta, S.: 1999, Managing advertising and promotion for long-run profability, Marketing Science 19(1), 1-22.

Kalbfleisch, J. and Prentice, R.: 1980, The Statistical Analysis of Failure Time Data, John Wiley \& Sons, New York.

Kamakura, W. A. and Russell, G. J.: 1989, A probabilistic choice model for market segmentation and elasticity structure, Journal of Marketing Research 26, 379-390.

Keane, M. P.: 1997, Modeling heterogeneity and state dependence in consumer choice behavior, Journal of Business \& Economic Statistics 15(3), 310-327.

Kiefer, N. M.: 1988, Economic duration data and hazard functions, Journal of Economic Literature 26, 646-679.

Lancaster, T.: 1979, Econometric methods for the duration of unemployment, Econometrica 47, 939-956.

Lancaster, T.: 1990, The Econometric Analysis of Transition Data, Vol. 17 of Econometric Society Monographs, Cambridge University Press, Cambridge. 
Mela, C. F., Gupta, S. and Lehmann, D.: 1997, The long-term impact of promotions and advertising on consumer brand choice, Journal of Marketing Research 34, 248-261.

Neslin, S. A., Henderson, C. and Quelch, J.: 1985, Consumer promotions and the acceleration of product purchases, Marketing Science 4(2), 147-165.

Paap, R. and Franses, P. H.: 2000, A dynamic multinomial probit model for brand choice with different long-run and short-run effects of marketing-mix variables, Journal of Applied Econometrics 15, 717-744.

Ridder, G.: 1990, The non-parametric identification of generalized accelerated failure-time models, Review of Economic Studies 57, 167-182.

Vakratsas, D. and Bass, F. M.: 2002, A segment-level hazard approach to studying household purchase timing decisions, Journal of Applied Econometrics 17, 49-59.

Vilcassim, N. J. and Jain, D. C.: 1991, Modeling purchase-timing and brand-switching behavior incorporating explanatory variables and unobserved heterogeneity, Journal of Marketing Research 28, 29-41. 